\title{
Analysis of the Impact of Hit Finding on Charge and Energy Reconstruction
}

Isabella Ginnett ${ }^{\mathrm{b}}$ advised by Minerba Betancourta and Bruce Howard ${ }^{\mathrm{a}}$

aFermi National Accelerator Laboratory, ${ }^{b}$ Michigan State University

\section{Introduction}

The ICARUS detector needs to accurately reconstruct particle interactions . to study interesting neutrino phenomena. Data reconstruction starts by processing wire plane signals into hits using a hit finder. The hits are used to calculate charge displaced per unit length, $d Q / d x$. Using $d Q / d x$, a calibration constant, and a charge to energy conversion formula, energy lost per unit length, $\mathrm{dE} / \mathrm{dx}$, is reconstructed.

Diagram of event reconstruction process in

CARUS [1]. Signals are measured by each of the

wire planes, converted into hits, and then use combined to construct tracks and showers.

\section{Study Objectives}

To investigate how different hit finders impact charge and $\mathrm{dE} / \mathrm{dx}$ reconstruction.

- To compare two absolute energy calibration techniques to determine the constants used to convert from the charge measured by the detector to displaced electrons.

\section{Hit Finder Study Methodology}

Utilizes samples of simulated muons and protons and three different hit finders, the Gauss, ICARUS raw, and hybrid hit finders.

- Gauss: deconvolve signals and fit to Gaussians.

- Raw: use raw wire plane signals and fit to an analytical function.

- Hybrid: input deconvolved signals into raw hit finder.

Hit finders are compared using plots of charge fractional difference, $Q_{\text {frac }}=\frac{Q_{\text {reco }}-Q_{\text {true }}}{Q_{\text {true }}}$. Checks for agreement between true and reconstructed charge

Plots of $d E / d x$ versus the residual range are created to compare the $\mathrm{dE} / \mathrm{dx}$ values calculated using a specific hit finder to theory.

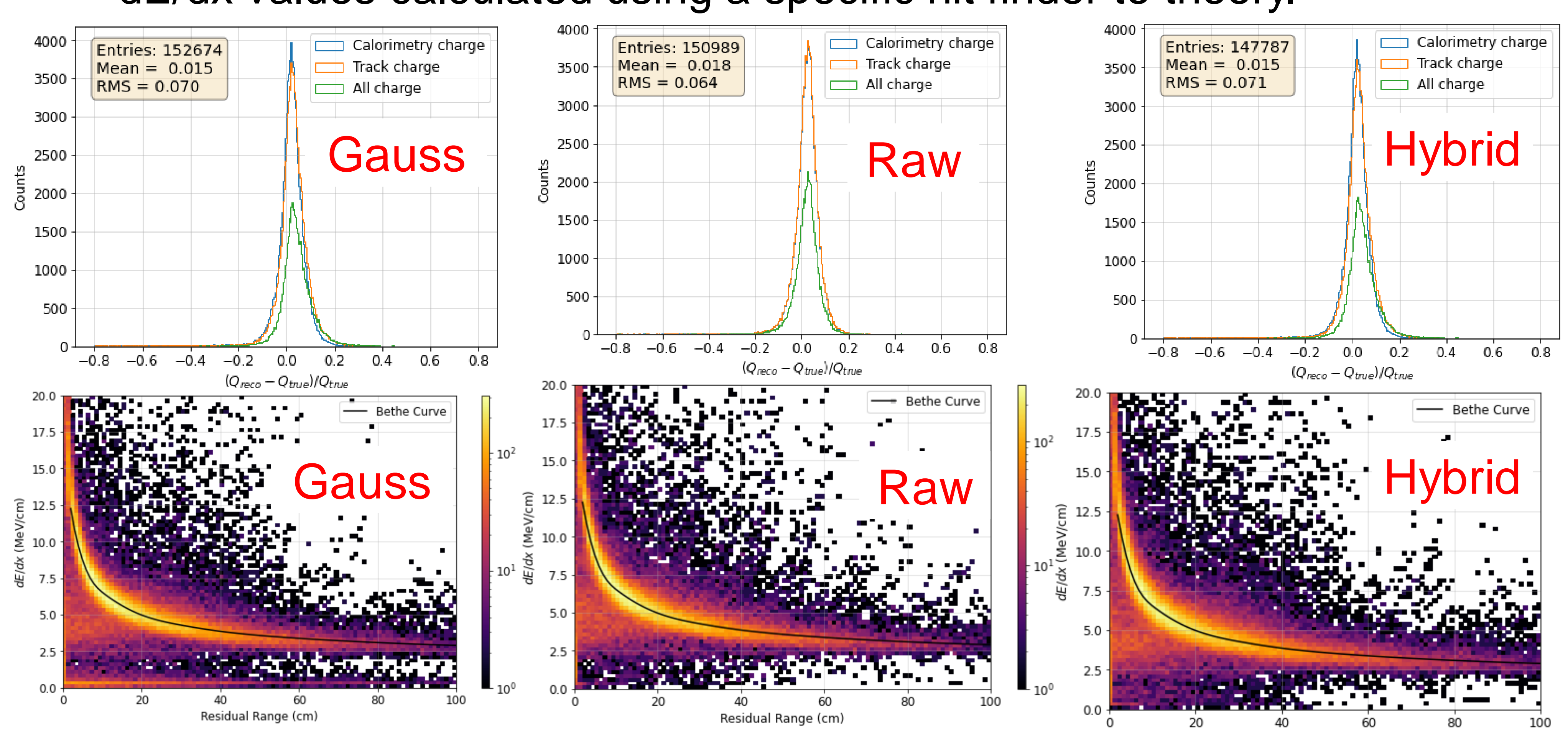

Charge fractional difference plots (top) and $\mathrm{dE} / \mathrm{dx}$ vs. residual range protots (bottom) for a proton sample on the collection plane of ICARUS
Absolute Energy Calibration Methodology

Utilizes samples of simulated muons in ICARUS and SBND that are well-confined and stopping in the detectors. Samples use the hybrid hit finder.

The MicroBooNE technique [2] uses the relationship between $\mathrm{dE} / \mathrm{dx}$ and $d Q / d x$.

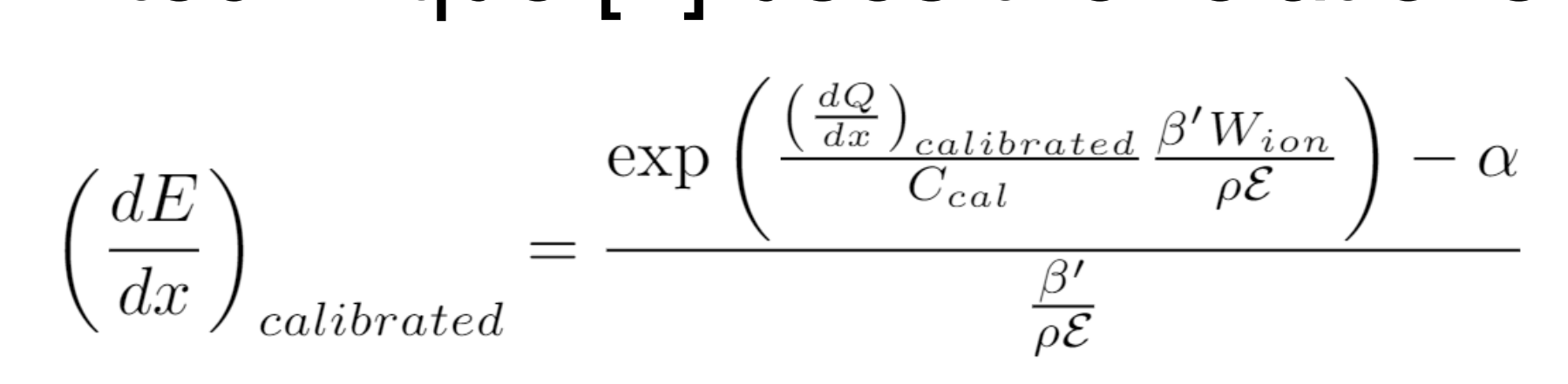

Corrected $\mathrm{dE} / \mathrm{dx}$ values are calculated and compared to theory using $\chi^{2}$ test, and the optimized constant is found by minimizing $\chi^{2} / n d f$

The LArIAT technique [3] also uses the relationship between $\mathrm{dQ} / \mathrm{dx}$ and $\mathrm{dE} / \mathrm{dx}$.

$$
\frac{d Q}{d x}=C_{c a l} \cdot \frac{d E / d x}{W_{\text {ion }}} \cdot R\left(\frac{d E}{d x}, \mathcal{E}\right)
$$

The $d Q / d x$ vs. $d E / d x$ curve is fit with the calibration constant as a fit parameter. The fit determines the optimized constant.

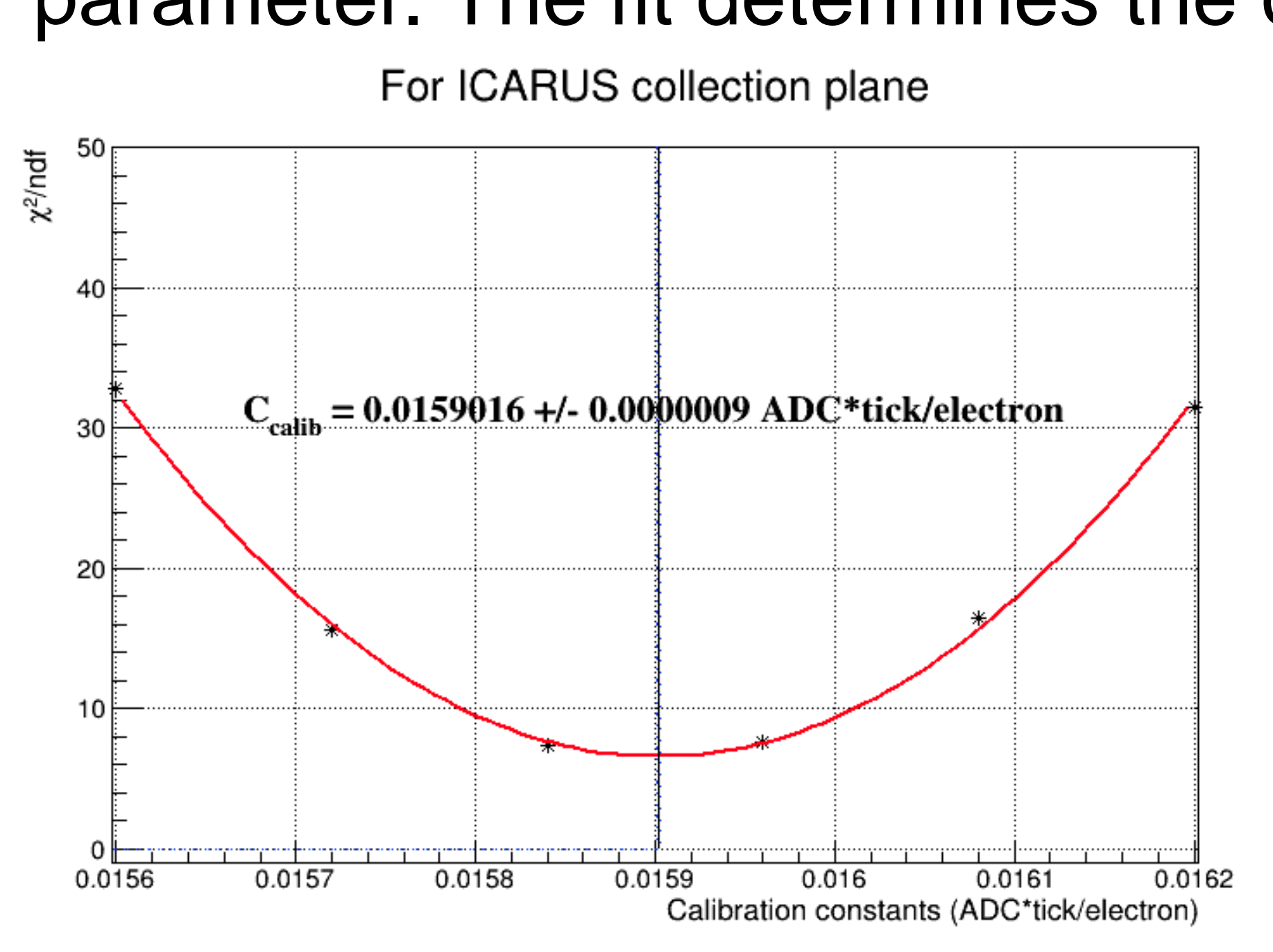

For ICARUS collection plane
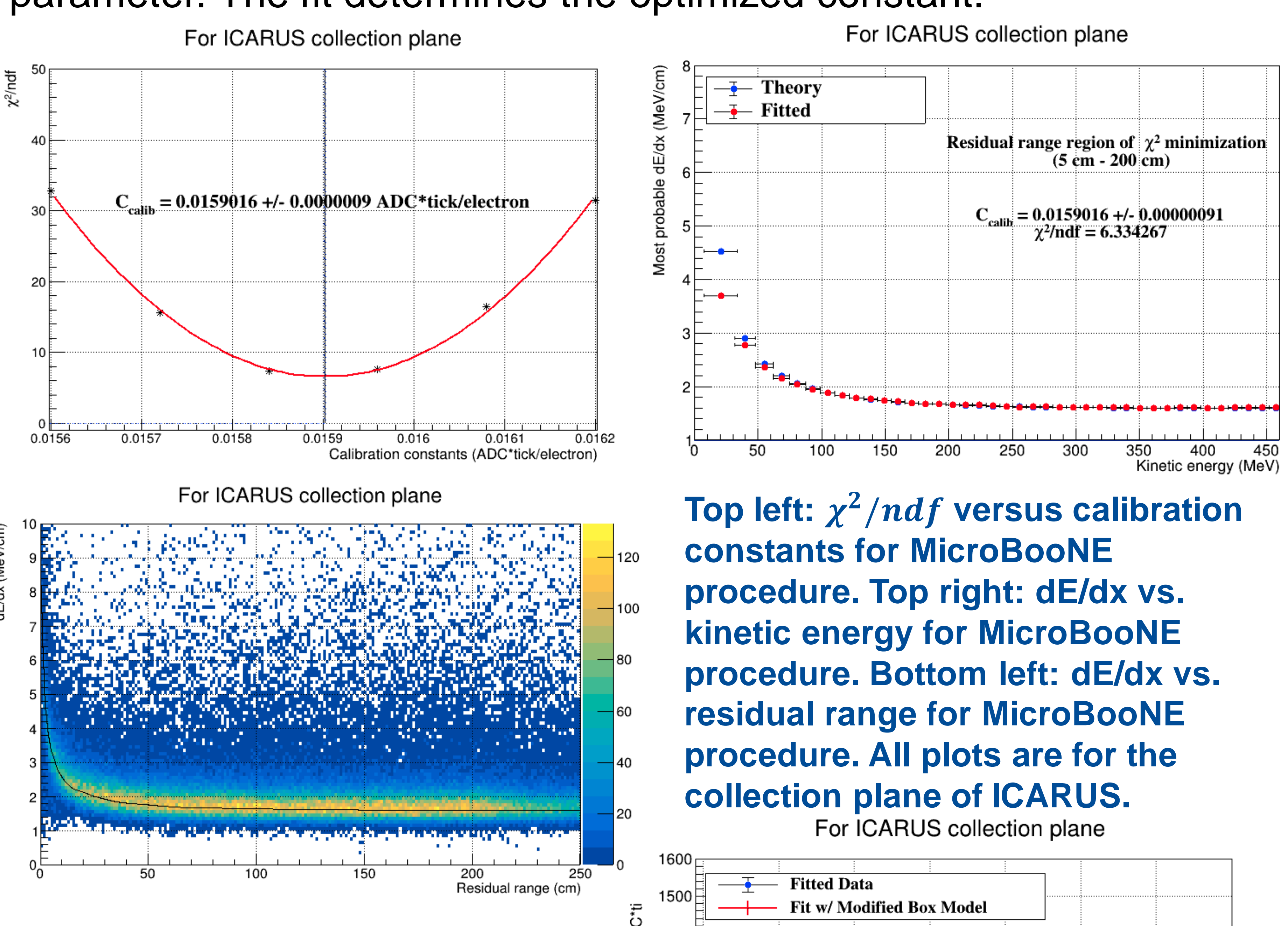

Top left: $\chi^{2} / n d f$ versus calibration

constants for MicroBooNE

procedure. Top right: $\mathrm{dE} / \mathrm{dx}$ vs.

kinetic energy for MicroBooNE

procedure. Bottom left: $\mathrm{dE} / \mathrm{dx}$ vs.

residual range for MicroBooNE

procedure. All plots are for the

collection plane of ICARUS.

Left: $d Q / d x$ vs. $d E / d x$ for LArIAT procedure. This plot is only for the collection

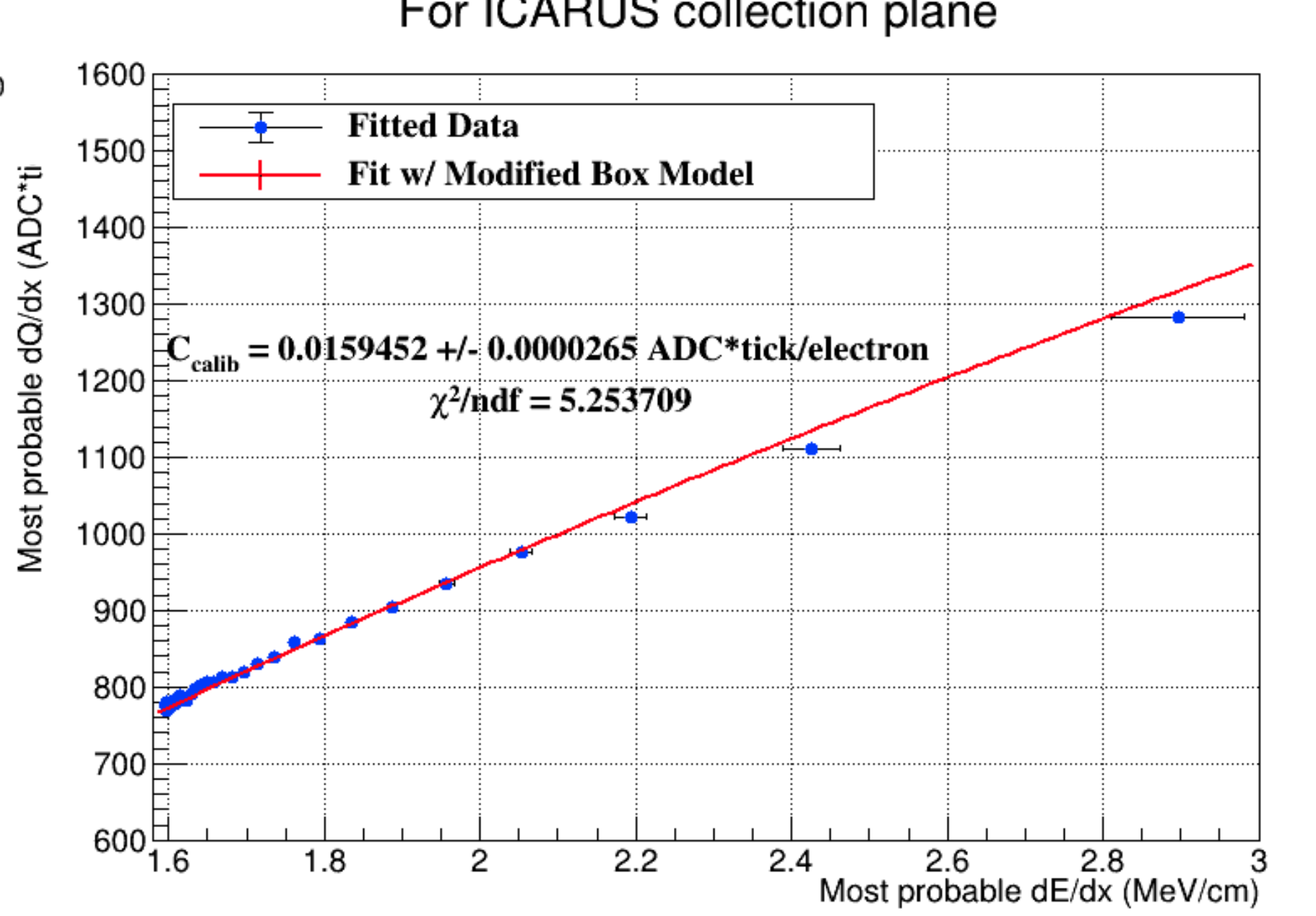

F MICHIGANSTATE
Table of calibration constants for ICARUS collection plane

MicroBooNE LArIAT

(ADC*tick/electron) (ADC*tick/electron)

$0.0159016+/-0.0000009^{*} 0.0159452+/-0.00003^{*}$

* Error bounds seem quite

small. Do they properly

Discussion of Results characterize calibration

All the charge fractional different plots are roughly centered at zero with a narrow distribution.

- The Gauss hit finder produced an excess of low $d E / d x$ values. This is from the Gauss hit finder being aggressive and splitting up long signals into many, small hits.

- The raw and hybrid hit finders perform better because of the Gauss signal splitting. More work is needed.

- The constants outputted from both techniques are similar and calibrate the data to correspond with theoretical expectations well.

- Next steps are to use a cosmic muon sample to further test calibration procedure and ultimately use a proton sample to check if there is agreement between the corrected data and theory.

\section{References}

[1] D. Schmitz and M. Bass, "Search for sterile neutrinos triples up", CERN Courier (2017)

2] C. Adams et al. (The MicroBooNE Collaboration), "Calibration of the charge and energy loss per unit length of the MicroBooNE liquid argon time projection chamber using muons and protons", arXiv:1907.11736, JINST 15, P03022 (2020).

3] R. Acciarri et al. (The LArIAT Collaboration), "The Liquid Argon In A Testbeam (LArIAT) Experiment”, arXiv:1911.10379, JINST 15, P04026 (2020).

\section{Acknowledgements}

I would like to thank my advisors Minerba Betancourt and Bruce Howard for their guidance, the ICARUS collaboration for providing the data and tools for the analysis, Fermilab for hosting, and the Department of Energy for funding. 